Revue internationale P.M.E.

Économie et gestion de la petite et moyenne entreprise

\title{
Évaluation de la performance et maîtrise du risque des entreprises industrielles françaises
}

\author{
Bernard Paranque, Dorothée Rivaud-Danset et Robert Salais
}

Volume 10, numéro 3-4, 1997

URI : https://id.erudit.org/iderudit/1009028ar

DOI : https://doi.org/10.7202/1009028ar

Aller au sommaire du numéro

\section{Éditeur(s)}

Presses de l’Université du Québec

ISSN

0776-5436 (imprimé)

1918-9699 (numérique)

Découvrir la revue

Citer cet article

Paranque, B., Rivaud-Danset, D. \& Salais, R. (1997). Évaluation de la performance et maîtrise du risque des entreprises industrielles françaises. Revue internationale P.M.E., 10(3-4), 11-38. https://doi.org/10.7202/1009028ar
Résumé de l'article

Pour un prêteur, l'appréciation qualitative des actifs d'une entreprise est une étape cruciale dans la prise de décision d'accorder ou non du financement. L'objet de cet article est de montrer que la difficulté de l'évaluation, source de rationnement des capitaux, vient de la diversité croissante des produits, des marchés et, par voie de conséquence, des actifs requis. Les méthodes généralement mobilisées ne nous permettent pas d'appréhender cette pluralité des situations économiques et financières. Les travaux empiriques menés reposent sur une classification ascendante hiérarchique réalisée sur un échantillon de PME françaises pour lesquelles on dispose d'informations quantitatives et qualitatives. Nous montrons qu'il y a plusieurs manières d'être rentable pour une entreprise, sans qu'il existe pour autant de critères autorisant à les hiérarchiser a priori. Les classes d'entreprises s'opposent par le marché auquel s'adresse le produit (générique ou dédié) et par /'organisation (actifs spécifiques ou standard). En revanche, la structure du capital et la rentabilité diffèrent peu d'une classe d'entreprises à l'autre. L’homogénéité obsen/ée au passif traduirait la difficulté des prêteurs à identifier les entreprises selon leur qualité, c'est-à-dire leur capacité à mettre en œuvre un "système d'action " que nous définissons comme un choix de produits et d'organisation qui soit cohérent et donc rentable. L 'article conclut à la nécessité d'une lecture de la performance qui reconnaisse cette diversité des systèmes d'action et valorise la cohérence de l'entreprise tant à l'interne qu'avec son environnement. Cette approche permet d'améliorer les capacités de prévision, et donc de gestion, du risque de crédit.
Ce document est protégé par la loi sur le droit d'auteur. L'utilisation des services d'Érudit (y compris la reproduction) est assujettie à sa politique d'utilisation que vous pouvez consulter en ligne.

https://apropos.erudit.org/fr/usagers/politique-dutilisation/ 


\title{
Évaluation de la performance et maîtrise du risque des entreprises industrielles françaises ${ }^{1}$
}

\author{
Bernard PARANQUE \\ Banque de France \\ Dorothée RIVAUD-DANSET \\ Université de Paris XIII
}

Robert SALAIS

IRESCO-CNRS

\section{MOTS CLÉS}

Structure du capital-Performance-Évaluation - Rentabilité

\section{RÉSUMÉ}

Pour un prêteur, l'appréciation qualitative des actifs d'une entreprise est une étape cruciale dans la prise de décision d'accorder ou non du financement. L'objet de cet article est de montrer que la difficulté de l'évaluation, source de rationnement des capitaux, vient de la diversité croissante des produits, des marchés et, par voie de conséquence, des actifs requis. Les méthodes généralement mobilisées ne nous permettent pas d'appréhender cette pluralité des situations économiques et financières. Les travaux empiriques menés reposent sur une classification ascendante hiérarchique réalisée sur un échantillon de PME françaises pour lesquelles on dispose d'informations quantitatives et qualitatives. Nous montrons qu'il y a plusieurs manières d'être rentable pour une entreprise, sans qu'il existe pour autant de critères autorisant à les hiérarchiser a priori. Les classes d'entreprises s'opposent par le marché auquel s'adresse le produit (générique ou dédié) et par l'organisation (actifs spécifiques ou standard). En revanche, la structure du capital et la rentabilité diffèrent peu d'une classe d'entreprises à l'autre. L'homogénéité observée au passif traduirait la difficulté des prêteurs à identifier les entreprises selon leur qualité, c'est-à-dire leur capacité à mettre en œuvre un "système d'action " que nous définissons

1. Étude réalisée en 1996 dans le cadre de la convention signée entre la Banque de France et le Groupement d'étude et de recherche IEPE-ENS Cachan, présentée au XLVe Congrès de l'Association française de sciences économiques et dont la version complète est publiée sous le titre «Marchés, organisations de la production et rentabilités », Banque de France B96/03. 
comme un choix de produits et d'organisation qui soit cohérent et donc rentable. L'article conclut à la nécessité d'une lecture de la performance qui reconnaisse cette diversité des systèmes d'action et valorise la cohérence de l'entreprise tant à l'interne qu'avec son environnement. Cette approche permet d'améliorer les capacités de prévision, et donc de gestion, du risque de crédit.

\begin{abstract}
When a lender receives a loan application from a company, the assessment of the company's assets is a crucial element in his decision to grant the financing. The purpose of this article is to demonstrate that difficulties in assessment, which can restrict access to capital, stem from the growing array of products, markets and, accordingly, the required assets. The methods generally applied are inadequate to apprehend the diversity of economic and financial situations. Our empirical research is based on a classification in ascending order of a sample of small and mediumsized French companies for which both quantitative and qualitative information was available. We show that there are several ways for a company to achieve profitability, but that in principle there are no criteria for establishing a hierarchical classification of the different methods. Companies are classified according to whether the markets for their products are generic or dedicated, and according to their organization, with either specific or standard assets. However, there is little difference between the capital structure and the profitability of companies in different classes. The uniform nature of companies' shareholders' equity reflects lenders' difficulty in assessing the creditworthiness of companies, that is, their ability to implement an «action system» defined as a coherent, and therefore profitable, choice of products and organization. The article concludes that assessments of corporate performances must take into account the diversity of action systems and emphasize both the internal coherence of a company and the way it relates to its environment. This approach leads to more precise forecasts, thereby improving the management of credit risks.
\end{abstract}

\title{
LES AUTEURS
}

Bernard Paranque, titulaire d'une habilitation à diriger les recherches en sciences économiques, est économiste à la Banque de France et chercheur au sein du département Monnaie Finance Banque de l'Université Lumière Lyon II. II travaille plus particulièrement sur les structures financières et le comportement des entreprises. Adresse : Banque de France, 44-1356, direction des Entreprises, 31, rue Croix-des-Petits-Champs, 75049 Paris Cedex 01, Tél. (33) 01 42925658 , email : frbdfxpq @ ibmmail-com.

Dorothée Rivaud-Danset est maître de conférences en sciences économiques à I'Université de Paris XIII. Elle travaille sur les relations entre la finance et l'industrie. Elle a publié sur ce sujet plusieurs articles dans des revues françaises. Adresse: Université de Paris XIII UFR de sciences économiques et de gestion, avenue Jean-Baptiste-Clément, 93430 Villetaneuse, Tél. (33) 0149403058.

Robert Salais est administrateur de l'INSEE et directeur de l'IRESCO (Institut de recherches sur les sociétés contemporaines-CNRS). Ses recherches portent sur l'économie du travail et sur la pluralité des formes de coordination économique. II a publié récemment plusieurs articles sur l'analyse économique des conventions et, avec Michael Storper (UCLA), un livre Les mondes de production. Enquête sur l'identité économique de la France, Éditions de l'EHESS, 1993. Adresse : IRESCO, 59-61, rue Pouchet, 75017 Paris, Tél. (33) 0140251061. 


\section{RESUMEN}

Para un prestamista, la apreciación de la calidad de los activos de una empresa constituye una etapa crucial en la decisión de concederle o no el financiamiento. El presente artículo tiene como objetivo demostrar que la dificultad de la evaluación, fuente de racionamiento de los capitales, proviene de la creciente variedad de los productos, de los mercados $y$, por consiguiente, de los activos requeridos. Los métodos generalmente utilizados resultan inadecuados para evaluar la diversidad de las situaciones económicas y financieras. Los trabajos empíricos realizados parten de una clasificación jerárquica ascendente realizada sobre una muestra de PyMEs francesas para las cuales disponemos de informaciones tanto cuantitativas como cualitativas. Demostramos que una empresa puede ser rentable de varias maneras, sin que ello suponga la existencia de criterios conducentes a establecer, a priori, una jerarquización. Los tipos de empresas se distinguen en función del mercado al que se dirige el producto (genérico o especial) y por la organización (activos específicos o estándar). Por el contrario, la estructura del capital y la rentabilidad difieren poco de un tipo de empresa a otro. La homogeneidad obsenada en el pasivo podría reflejar la dificultad de los prestamistas para identificar las empresas según su calidad, es decir, su capacidad de poner en marcha un «sistema de acción" que definimos como una selección de productos y de organización coherente $y$, por ende, rentable. El artículo concluye refiriéndose a la necesidad de una interpretación de la actuación de la empresa, que reconozca la diversidad de los sistemas de acción y valore la coherencia de la empresa, tanto internamente como en relación a su entorno. Este enfoque permite mejorar las capacidades de previsión y, por tanto, de gestión del riesgo del crédito.

\section{Introduction}

L'appréciation de la qualité des entreprises est un problème essentiel du financement de l'économie. La difficulté d'évaluer et, en conséquence, d'apporter des capitaux à une entreprise vient, selon nous, de ce que la diversité croissante des biens et services produits et des actifs nécessaires à leur production bute sur l'unicité des méthodes, sur leur caractère trop statique et immédiatement financier. Nous voulons montrer a contrario l'importance qu'il y aurait à développer le caractère pluriel, dynamique et « réel » de l'évaluation financière. Cette voie de recherche s'inspire des analyses récentes de la diversité des produits en termes de pluralité des mondes de production (Salais et Storper, 1993), où les produits se caractérisent par la nature du marché visé et par l'organisation de la production. Le marché visé peut être prévisible ou incertain, diversifié ou générique, tandis que les technologies requises et la main-d'œuvre employée, qualifiant l'organisation de la production, peuvent être standard ou spécifiques. De la pluralité de ces mondes se déduit l'existence de plusieurs modèles de formation de la rentabilité qui décomposent le taux de rentabilité en différents ratios usuels. Ces modèles traduisent les contraintes « réelles », inscrites dans le produit, que doivent gérer les acteurs (attentes des clients, nature 
prévisible ou incertaine du marché, technologie standard ou spécialisée, importance des savoir-faire, etc.). Pour désigner l'existence de différentes manières d'être rentable, nous parlerons de système d'action. Un système d'action représente une des cohérences praticables et, donc, rentables pour une entreprise. Il prend en compte la spécificité des contraintes qui pèsent sur l'entreprise. Il existe différents systèmes d'action, mais il n'y a aucun critère autorisant à hiérarchiser leurs performances.

Pour les tiers, apporteurs externes de capitaux, ce qui importe, c'est la qualité des entreprises ; la qualité étant entendue non pas au sens commun des caractéristiques économiques et financières, mais au sens de la capacité des entreprises à mettre en œuvre un système d'action, donc à respecter une des cohérences rentables. L'intérêt de disposer d'une pluralité de grilles d'évaluation de la qualité des entreprises se déduit de cette recherche.

Le travail empirique présenté identifie la pluralité des systèmes d'action et des qualités des entreprises illustrée par la diversité de positionnement des entreprises. Ce constat est interprété en référence à la pluralité des mondes de production. L'étude des ratios contribuant à la formation de la rentabilité économique montre ensuite la diversité des modèles de gestion de la rentabilité. Elle est suivie par le repérage statistique de plusieurs classes d'entreprises. Enfin, la théorie économique permet d'éclairer le constat d'une relative homogénéité de la structure du capital et de la rentabilité d'une classe à l'autre. La conclusion s'intéresse au problème de l'évaluation de la qualité de l'entreprise par les tiers. Elle souligne l'importance, pour la banque notamment, de l'intégration d'informations qualitatives pertinentes pour évaluer la qualité des entreprises ${ }^{2}$.

\section{Une grande variété d'entreprises}

La recherche présentée ici se préoccupe de saisir la cohérence de la firme qui se construit autour du produit, non pas au sens du secteur d'activité, mais en référence au marché et à l'organisation de la production, comme le précise l'introduction. Dans cette perspective, elle utilise un matériau original, l'enquête dite SÉSAME ${ }^{3}$, qui fournit une base de données qualitatives.

2. Le lecteur intéressé trouvera les résultats détaillés de cette recherche dans Paranque, Rivaud-Danset et Salais (1996).

3. SÉSAME : Système élargi de support multicritère des entreprises. 


\section{ENCADRÉ 1}

\section{Présentation de l'échantillon et de la base de données SÉSAME}

Depuis 1993, une base de données qualitatives est constituée à partir d'enquêtes directes auprès de chefs d'entreprise volontaires, à l'aide d'un questionnaire spécifique assisté par ordinateur. Deux cents questions permettent de cerner, d'une part, les caractéristiques de l'environnement au sens large (clients, fournisseurs, nouveaux entrants, produits de substitution, rivalité interne) et, d'autre part, les orientations stratégiques par activité (positionnement, objectifs, avantages concurrentiels recherchés et fonctions privilégiées dans la poursuite de ces objectifs). À cette date, pour la première fois, 2000 chefs d'entreprise des secteurs des biens d'équipement professionnel et des biens intermédiaires ayant moins de 2000 employés ont répondu à un questionnaire, lors d'entretiens approfondis d'une durée moyenne de deux heures et demie. Seules 1354 entreprises sont adhérentes à la Centrale de bilans de la Banque de France et présentes, de manière continue de 1991 à 1993, dans notre échantillon. Le questionnaire SÉSAME donne, comme indicateurs utilisables pour caractériser les entreprises en fonction de leurs produits, les éléments suivants :

- la nature du processus de production (tâches répétitives ou non);

- la qualité du produit (haut ou bas de gamme);

- le degré de standardisation du produit (élevé ou faible);

- le mode de compétitivité dans lequel l'entreprise est inscrite (fondée sur la différenciation de l'offre ou sur le prix);

- la relation avec la demande selon sa nature supposée (fabrication en grande série destinée à une demande indifférenciée, fabrication à l'unité pour une demande individualisée);

- le mode de commercialisation (sept modes possibles);

- la dépendanice du client envers l'entreprise (coût élevé ou négligeable de recherche d'un autre fournisseur si l'entreprise fait défaut);

L'importance des dépenses immatérielles (recherche-développement, marketing). L'analyse de données à partir du questionnaire SÉSAME permet de relever des différences de positionnement entre entreprises se traduisant par des cohérences spécifiques d'organisation et de présence sur le marché. Celles-ci sont mises en rapport avec l'analyse de ratios financiers caractéristiques de la formation de la rentabilité. Le critère des effectifs autorise à qualifier de PME les entreprises de l'échantillon. Il a l'avantage de la simplicité mais l'inconvénient de l'arbitraire. Cependant, il ressort de cette étude que la taille des entreprises appréhendée à travers le nombre de salariés occupés ne constitue par un déterminant important des classes définies par les différents types de cohérence « organisation ou marché». 
Cette enquête permet de relever des différences de positionnement entre entreprises, les questions portant sur les caractéristiques de l'environnement au sens large (des clients aux produits de substitution) et sur les orientations stratégiques.

Trente-cinq questions de l'enquête 1993, seule année disponible au moment de la recherche, permettent d'identifier les principales caractéristiques concernant le positionnement des entreprises sur leur marché, douze autres questions et huit ratios financiers viennent compléter cette étude.

L'analyse de données est réalisée sur 1354 entreprises. Les vingt premiers axes de l'analyse en composantes principales (ACP) expriment 78,5\% de l'inertie totale.

\section{ENCADRÉ 2}

Les 1354 sociétés étudiées peuvent être représentées comme constituant un nuage de points dans un espace à 35 dimensions correspondant aux 35 questions. Il s'agit alors de trouver, après transformation du tableau de départ, l'ensemble de droites ou d'axes principaux linéairement indépendants qui donnent la meilleure représentation possible du nuage initial. L'analyse en composantes principales calcule les axes principaux en utilisant un algorithme qui maximise la somme des carrés des distances entre les projections orthogonales des points et le centre de gravité du nuage, point commun de toutes les droites cherchées. Ainsi, l'ACP permet de réduire l'information de base disponible sur chaque individu et de construire des facteurs synthétiques (indicateurs), appelés aussi axes.

L'identification des différences de positionnement reflétant divers types de cohérences « organisation ou marché » entre les entreprises s'effectue à partir des cinq premiers axes :

- axe 1 : les sociétés du côté positif de l'axe, travaillant sur des petites séries, soucieuses des attentes de leurs clients, positionnées sur le haut de gamme, s'opposent à celles dont l'activité est fortement standardisée et qui produisent en grandes séries;

- axe 2 : les sociétés ayant une compétitivité fondée sur les prix se distinguent de celles se différenciant par leur offre, positionnées du côté positif de l'axe ;

- axe 3 : les entreprises travaillant en grandes séries et / ou dont le chiffre d'affaires est réalisé avec peu de clients se distinguent de celles produisant à l'unité ;

- axes 4 et 5 : ces axes complètent et précisent les précédentes différenciations avec la distinction chiffre d'affaires haut de gamme ou milieu et bas de gamme et une explicitation des modes de distribution. 
Le graphique présente les axes 1 et 2 de l'analyse factorielle et permet une première visualisation de ces positionnements ${ }^{4}$.

\section{GRAPHIQUE 1}

\section{Projection de variables sur les deux premiers axes de l'analyse factorielle et représentation du cercle des corrélations}

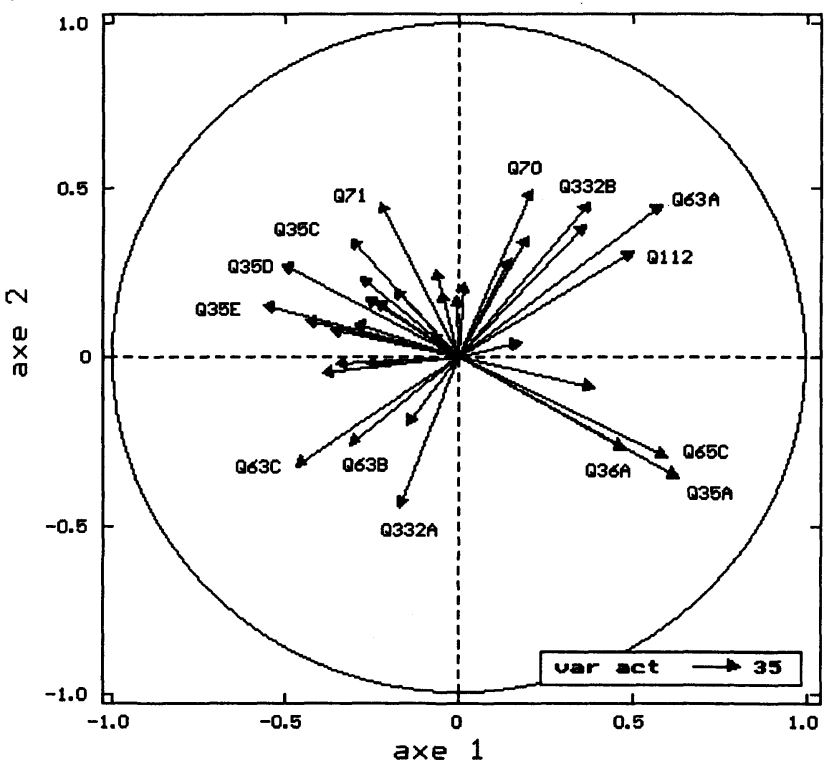

Légende :

Q332A : rôle du prix dans la décision d'achat

Q332B : rôle de l'originalité ou de la supériorité de l'offre dans la décision d'achat des clients

Q35A : faible standardisation du produit

Q35C : produits concernés par une production en moyenne série

Q35D : standardisation assez forte

Q35E : forte standardisation

Q36A : produits concernés par une production à l'unité
Q63 A : part du chiffre d'affaires réalisée en haut de gamme

Q63 B : part du chiffre d'affaires réalisée en milieu de gamme

Q63C : part du chiffre d'affaires réalisée en bas de gamme

Q65 C : part du chiffre d'affaires réalisée par l'intermédiaire des filiales et établissements

Q70 : part de la recherche et développement dans le chiffre d'affaires

Q71 : part des dépenses marketing et publicité dans le chiffre d'affaires

Q112 : degré de spécificité des attentes clients

Source: Banque de France.

4. Chaque axe a un pouvoir explicatif spécifique; en particulier, les valeurs propres des deux premiers axes sont bien distinctes. Cependant, le fait que les cinq premiers axes de l'ACP n'expriment que $35 \%$ de l'inertie totale mérite d'être commenté. L'éventail des positionnements des entreprises ne se réduit pas à un nombre a priori limité de stratégies. Une seconde explication non contradictoire peut être avancée. La perception du positionnement de l'entreprise est sans doute loin d'être évidente pour tous les chefs d'entreprise interrogés, ce qui les inciterait à fournir des réponses non discriminantes à certaines questions (le questionnaire prévoit souvent un large éventail de réponses et donc la possibilité de se situer dans une zone moyenne). 
Après avoir défini les deux principales dimensions de l'espace occupé par les entreprises et leurs réponses, on relève que le premier axe de l'analyse factorielle rend compte de l'organisation de la production et le deuxième axe renvoie aux déterminants de la compétitivité, donc aux relations de l'entreprise avec le marché. L'opposition entre l'organisation et le marché qui apparaît ainsi sur ces deux premiers axes rend ces derniers assez proches des axes théoriques retenus par Salais et Storper dont la conjonction définit les mondes possibles de production (Salais et Storper, 1993), comme permet de le visualiser le graphique 2.

GRAPHIQUE 2

Rapprochement des mondes théoriques et de l'analyse empirique

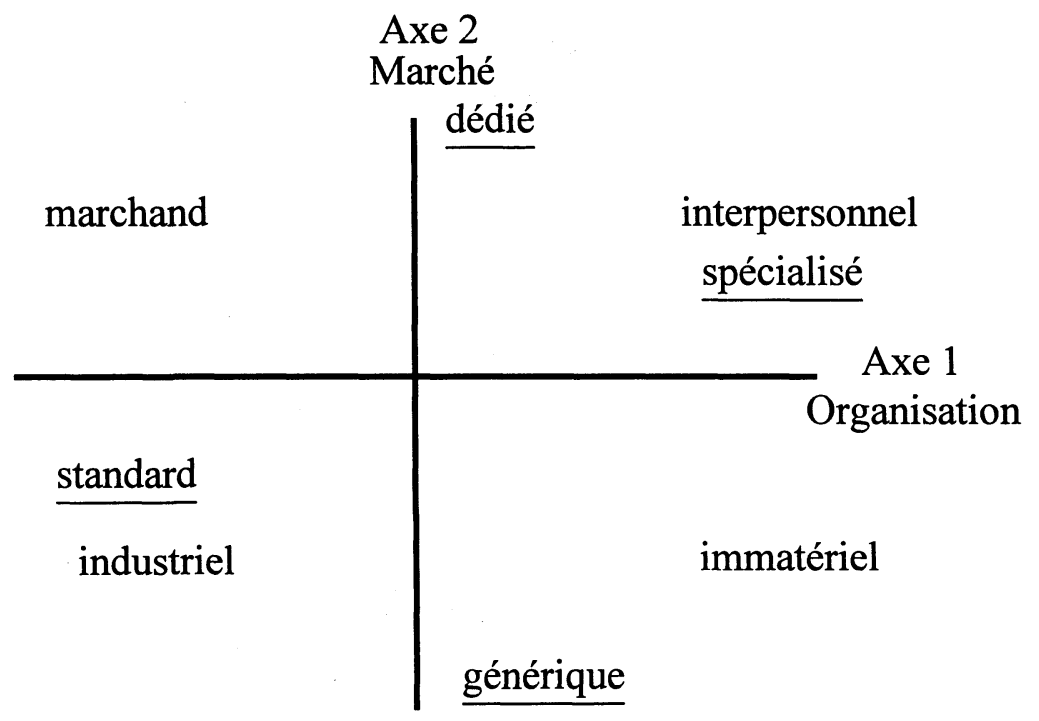

Ces mondes de production sont structurés le long du premier axe selon l'opposition entre un principe de standardisation du produit (des technologies connues et diffusées permettent une production en grande série) et un principe de spécialisation du producteur (des savoir-faire et des technologies spécifiques à l'entreprise permettent de produire en petite série). Ils se trouvent également structurés selon une seconde opposition, celle des relations de l'entreprise avec son marché. Celle-ci sépare le produit générique, c'est-à-dire le produit dont la définition est sous la seule responsabilité de l'offreur et qui est conçu pour une demande indifférenciée, et le produit dédié à des segments de demande ou à des demandes particulières intégrant, dans ce cas, les besoins particuliers du demandeur dans 
l'optique d'un meilleur service. La conjonction des deux oppositions définit quatre mondes de production ayant une cohérence propre, multidimensionnelle, tentant d'intégrer les contraintes dérivées des modes d'organisation de la production praticables et des attentes des demandeurs et des marchés.

Dans la partie ouest du graphique, on retrouve des entreprises réalisant des produits à dominante standard, dont l'offre se différencierait en fonction des spécifications de la demande à mesure que l'on «monte » vers le quadrant nord-ouest (autrement dit, vers des produits «marchands») ou, au contraire, deviendrait de plus en plus générique ${ }^{5}$ (vers des produits qu'il serait naturel d'appeler « industriels») à mesure que l'on « descend » vers le quadrant sud-ouest. La partie est du graphique se caractériserait, en revanche, par des entreprises réalisant des produits à dominante spécialisée, dont la dédicace à des demandes individuelles spécifiques croîtrait à mesure que l'on monte vers le quadrant nord-est (vers des produits qu'il est suggéré pour ces raisons d'appeler « interpersonnels » selon Salais et Storper) et diminuerait à mesure que l'on redescend vers le quadrant sud-est (produits à composante immatérielle grandissante).

\section{La rentabilité : une norme accessible selon plusieurs modèles}

Le recours à une typologie permettra d'illustrer la thèse selon laquelle il y a plusieurs façons d'être rentable. Avant d'y procéder, il importe de distinguer les ratios que nous qualifierons de synthétiques et les ratios que nous dénommerons interprétatifs.

Les ratios synthétiques peuvent $s$ 'analyser comme des normes ou des forces de rappel dont on ne peut s'écarter durablement. On attend des ratios synthétiques qu'ils admettent une valeur qui différencie peu les classes d'entreprises ( $c f$. infra). Cette valeur moyenne exprime la cohérence du positionnement des entreprises et non un simple résultat statistique. Il s'agit des ratios de taux de marge et de rentabilité économique.

Il existe deux modes de calcul du taux de marge : l'un rapporte l'excédent brut d'exploitation à la valeur ajoutée, l'autre au chiffre d'affaires. Dans le premier cas, le taux de marge (EBE/VA) exprime les possibilités de valorisation des produits de l'entreprise. Il peut s'analyser aussi dans une perspective de partage de la valeur ajoutée, comme le complément à l'unité de la part des salaires dans la valeur ajoutée (WN/VA, W étant la rémunération des salariés et $\mathrm{N}$, l'effectif). Il dépend alors du taux de rémunération et de la productivité apparente du travail, analysée comme un rendement. Dans le deuxième cas (EBE/CA), l'optique est plutôt commerciale et entend rendre compte des relations avec le marché. On peut exprimer de

5. Produits dont la définition est sous la responsabilité de l'offreur. 
manière classique EBE/CA sous la forme [(VA/CA) $\times(\mathrm{EBE} / \mathrm{VA})]$. Cette décomposition, qui fait apparaître le taux de valeur ajoutée, montre que le taux de marge dépend, en outre, d'une série de déterminants : le taux d'intégration (qui renvoie aux techniques de production), l'organisation de la production, l'efficience dans l'usage des consommations intermédiaires et, enfin, les prix relatifs qui résultent des relations avec les clients et les fournisseurs. Ce dernier déterminant dépend, lui-même, de la capacité à intégrer dans le prix la qualité du produit telle que nous l'étudions ici, c'est-à-dire en référence au couple organisation de la production et marché. Deux entreprises peuvent dégager ainsi un taux de marge identique, selon deux principes distincts : une valorisation élevée du produit peut neutraliser le poids de la masse salariale dans la valeur ajoutée, de même qu'une productivité apparente du travail élevée, accompagnée d'un taux de rémunération moyen, peut compenser une moindre possibilité de valoriser le produit.

Les ratios interprétatifs, comme le coût unitaire de la main-d'œuvre, l'intensité capitalistique et le poids des besoins en fonds de roulement, rendent compte du principe d'optimisation ou de recherche d'un compromis satisfaisant adopté par l'entreprise pour être rentable. Lorsqu'on décompose les déterminants de la rentabilité en une batterie de ratios, ce n'est pas l'optimisation de chacun de ces ratios qui est ou devrait être recherchée, mais l'optimisation du « système réel » exprimant la cohérence de l'entreprise dans son organisation interne et dans ses relations avec son environnement. L'interprétation de ces ratios n'a de sens qu'en s'inscrivant dans une des décompositions possibles. Étudiés isolément, ces ratios sont souvent ambigus. Ainsi, l'importance des délais accordés aux clients ou par les fournisseurs peut, en dehors des usages de la profession, exprimer le souci de pérenniser la relation de l'entreprise avec ses clients et ses fournisseurs ou traduire des difficultés. De même, le coût unitaire de la main-d'œuvre ne peut suffire à exprimer la spécificité des compétences déployées par les salariés de l'entreprise, la relation entre rémunération et spécialisation supposant que les qualifications soient reconnues sur le marché du travail.

La rentabilité est alors étudiée à partir de décompositions qui mettent l'accent soit sur le marché (taux de rotation des capacités productives...), soit sur le procès de production (productivité des facteurs, intensité capitalistique).

La première décomposition insiste sur une recherche de la rentabilité guidée par le marché, c'est-à-dire sur l'ajustement dans le court terme des actifs à la demande. Soit, en reprenant les notations usuelles :

EBE : excédent brut d'exploitation

$\mathrm{K}$ : capital (actif immobilisé brut)

AC - PC : actif circulant - passif circulant (besoins en fonds de roulement)

$\mathrm{N}: \quad$ effectif 
CA: chiffre d'affaires

W: coût unitaire de la main-d'œuvre

$[1] \mathrm{EBE} /[\mathrm{K}+(\mathrm{AC}-\mathrm{PC})]=\frac{\mathrm{EBE} / \mathrm{CA}}{(\mathrm{K} / \mathrm{CA})+[(\mathrm{AC}-\mathrm{PC}) / \mathrm{CA}]}$

L'objectif de rentabilité est atteint en minimisant le dénominateur, ce qui est obtenu par la rotation rapide des actifs fixes et circulants. En donnant la priorité aux flux, l'entreprise limite ses actifs, d'où une intensité capitalistique moindre.

La seconde décomposition met l'accent sur l'organisation de la production dont l'efficacité s'exprimerait par le critère de la productivité du travail :

[2] $\mathrm{EBE} /[\mathrm{K}+(\mathrm{AC}-\mathrm{PC})]=\left[1-\frac{\mathrm{WN}}{\mathrm{VA}}\right] \times \frac{\mathrm{VA} / \mathrm{N}}{(\mathrm{K} / \mathrm{N}+[(\mathrm{AC}-\mathrm{PC}) / \mathrm{N}]}$

Les entreprises donnent la priorité à la maximisation de la productivité du travail, ce qui suppose d'accepter d'immobiliser davantage les actifs fixes, d'avoir une intensité capitalistique supérieure et une moindre efficacité du capital. Le fait de mettre l'accent sur une organisation de la production qui donne la priorité à l'efficacité des ressources immobilisées (ici la main-d'œuvre) suppose d'accepter un taux de rotation des actifs circulants moins élevé. Deux interprétations du poids des actifs circulants sont alors possibles :

- le marché est prévisible, la firme traite les fluctuations de la demande comme si elles se compensaient autour d'une tendance centrale et connue; dans cette situation, la gestion des stocks lisse les fluctuations ;

- la firme entend construire des relations de clientèle durables et accepte des délais clients relativement longs.

\section{La formation de la rentabilité économique selon les classes d'entreprises}

Les différences de positionnement des entreprises, mises en évidence précédemment, permettent d'identifier cinq classes. Ces classes témoignent d'une diversité concrète des modèles d'organisation plus grande que ne le suppose le point de vue normatif usuel. Cette diversité conforte celle observée sur les comportements économiques des entreprises (Bardos et Paranque, 1992; Paranque, 1995). Elles expriment qu'il existe des systèmes d'action différents pour les entreprises et que ces derniers sont adaptés à des ensembles de contraintes spécifiques (attentes des clients, nature prévisible ou incertaine du marché, technologie standard ou spécialisée, importance des savoir-faire, etc.). La spécificité de leur produit en résulte, ainsi que celle des problèmes que les firmes ont à résoudre pour demeurer rentables.

Avant de présenter les classes d'entreprises, quelques remarques sont nécessaires à la compréhension des résultats. En particulier, il faut relever que les 
entreprises disposent d'un éventail de positionnement plus grand que celui auquel on pouvait s'attendre et non d'un choix limité, composé de quelques stratégies clairement identifiables a priori. On constate, par exemple, que les entreprises qui font du haut de gamme et des produits standardisés se distinguent de celles qui fabriquent également en haut de gamme, mais des produits sur mesure, ce qui rappelle que haut de gamme n'est pas synonyme de sur mesure et inversement.

La taille est une variable parfois discriminante, mais moins nettement que ce que l'on suppose habituellement, ce qui conduit à s'interroger sur la pertinence des critères habituellement retenus pour distinguer grandes entreprises et PME. En effet, on retrouve une opposition, d'une part sur l'axe 1 entre sociétés employant entre 100 et 500 personnes du côté négatif et les petites entreprises de moins de 100 salariés, d'autre part, sur l'axe 2, entre ces dernières (côté négatif) et les entreprises de plus de 500 employés. En outre, l'examen de la répartition des entreprises dans les classes selon leur taille montre une certaine indépendance entre ces deux variables, ce qui permet de considérer que l'importance des effectifs a peu d'influence sur l'appartenance à tel ou tel regroupement. Ainsi, l'absence de référence à ce critère dans la description de certaines classes ne fait que refléter ce constat.

GRAPHIQUE 3

Rentabilité brute globale

(en pourcentage)

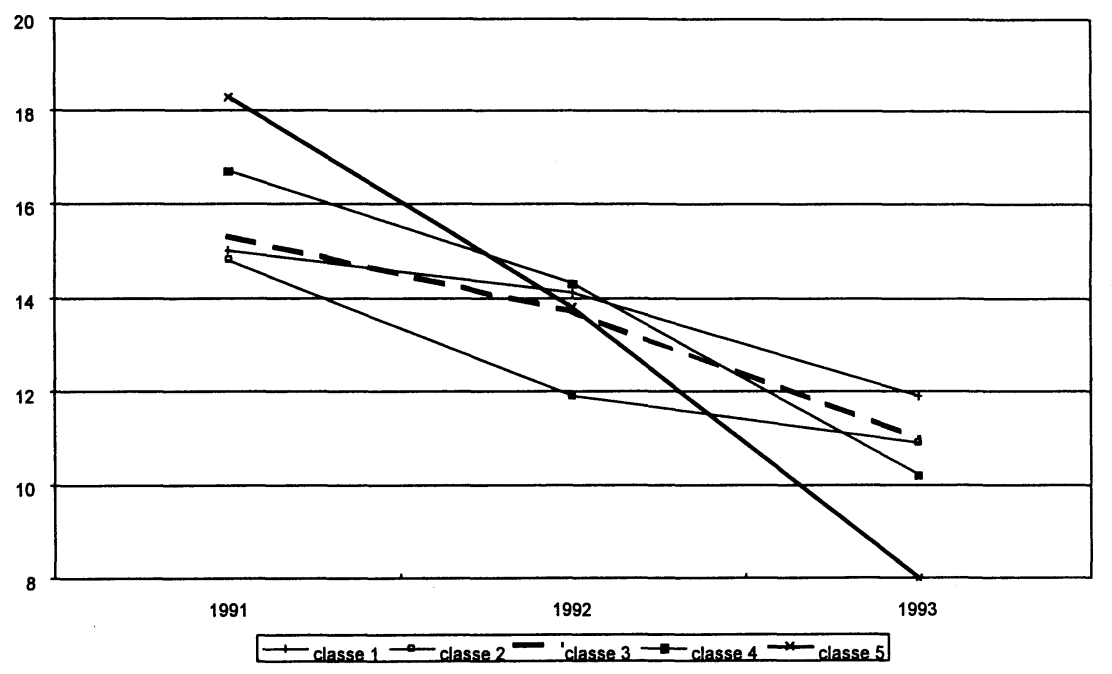

Source: Banque de France. 
Les ratios de rentabilité économique ${ }^{6}$ et de taux de marge admettent des valeurs moyennes assez proches, du moins pour les quatre premières classes. Ce résultat est robuste de 1991 à 1993, hormis pour la classe 5 qui, plus rentable en 1991 et 1992, « décroche » brutalement en 1993. Il n'y a donc pas de hiérarchie dans les performances entre ces systèmes d'action. Ce résultat se retrouve à propos de la rentabilité financière nette, ce qui indique une neutralité de la fiscalité et des prélèvements financiers sur les «qualités » des entreprises. Il renforce l'idée selon laquelle il peut exister plusieurs modèles de production profitables, à condition qu'ils soient mis en œuvre de façon cohérente.

\section{TABLEAU 1}

\section{Écart des déterminants de la rentabilité selon la classe Par rapport à la moyenne de l'échantillon ${ }^{a}$}

\begin{tabular}{lccccc}
\hline Classes & $\mathbf{1}$ & $\mathbf{2}$ & $\mathbf{3}$ & $\mathbf{4}$ & $\mathbf{5}$ \\
\hline Rentabilité économique $^{b}$ & ++ & ++ & ++ & + & -- \\
VAG/ capital engagé $*$ & - & - & - & - & ++ \\
VAG/ frais de personnel & + & + & + & - & -- \\
Capital engagé / effectifs & - & + & ++ & + & -- \\
VAG/ effectifs & - & ++ & ++ & - & - \\
Excédent brut global / production & + & + & + & + & -- \\
Besoins en fonds de roulement / production & + & ++ & + & + & -- \\
Production / équipement productif & ++ & + & - & -- & ++ \\
Frais de personnel/effectif & -- & ++ & - & - & + \\
\hline
\end{tabular}

$a$ Le signe + indique un écart positif, le signe - un écart négatif ; le doublement du signe exprime l'ampleur. $b$ VAG : valeur ajoutée Centrale de bilans + produits financiers + charges exceptionnelles nettes.

Source: Banque de France.

Les deux modèles proposés de formation de la rentabilité ne prétendent pas rendre compte de l'ensemble des combinaisons de ratios possibles et des principes qui les guident. Ainsi, l'on pourrait concevoir un modèle où serait maximisée l'efficacité du capital (Salais et Storper, 1993 ; Paranque, 1994). Néanmoins, pour certaines classes, les combinaisons de ratios retenues peuvent offrir une description approchée

6. Excédent brut global/ capital engagé :

- excédent brut global $=\mathrm{EBE}+$ produits financiers + charges exceptionnelles nettes ;

- capital engagé $=$ financement propre + endettement financier $=$ actif immobilisé brut + besoins en fonds de roulement ;

- financement propre $=$ capitaux propres + réserves d'autofinancement (amortissements et provisions). 
des modèles théoriques. C'est ainsi que nous avons conduit des tests d'inégalité de moyenne sur les ratios entre les classes ${ }^{7}$.

À partir des résultats de la classification et de la projection des classes sur les deux premiers axes de l'analyse de données (graphique 4), il est possible de préciser les profils de ces groupes d'entreprises.

GRAPHIQUe 4

Projection des classes d'entreprises

sur les deux premiers axes de l'analyse factorielle

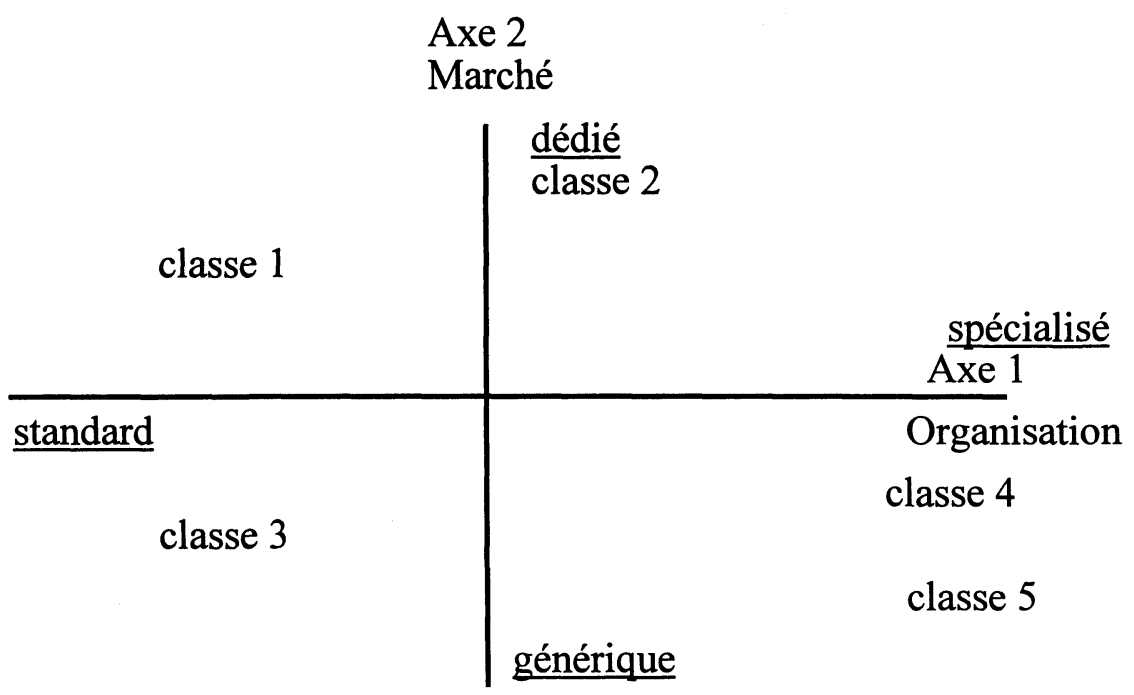

Classe 1 (9,8\% des entreprises) :

produits standard pour le marché final

La classe 1 réunit des entreprises dont les débouchés sont essentiellement constitués par le marché final des particuliers. Le produit est fabriqué plutôt en grande série et assez fortement standardisé. Les dépenses de marketing sont relativement importantes. Ces firmes sont plutôt dans le quadrant nord-ouest du graphique 2 ; elles peuvent être considérées comme étant proches du modèle de rentabilité dit

7. Voir l'annexe. La moyenne de chaque classe est présentée avec son intervalle de confiance au seuil de $5 \%$ pour faciliter la lecture des écarts. 
«marchand». Les effets d'une productivité du travail peu élevée, a priori peu favorables à la rentabilité, sont plus que compensés par une rémunération du travail et une intensité capitalistique inférieures à la moyenne générale. La bonne rentabilité globale est obtenue par des taux de rotation des équipements et de l'actif circulant élevés, en raison, pour ce dernier, des faibles délais de règlement fournisseurs et clients. Le positionnement de ces entreprises implique une flexibilité et donc une limitation des engagements en capital fixe et circulant afin de les ajuster dans le court terme à la demande. Enfin, aucune taille d'entreprises ou activité n'apparaissait de manière prépondérante; on retrouve ici la même proportion de petites PME, de PME, de grandes PME et de grandes entreprises que sur l'ensemble de l'échantillon.

\section{Classe 2 (18,5\% des entreprises): produits dédiés à des usages spécifiques}

La classe 2 groupe des sociétés qui produisent en petites séries et dont la compétitivité repose principalement sur une différenciation par l'offre. Bien que leurs produits puissent être standardisés, ils se positionnent en haut de gamme et se distinguent par la technologie. La part du chiffre d'affaires consacrée à la recherchedéveloppement, aux dépenses de marketing et de publicité est plus élevée que pour l'ensemble de l'échantillon. Les entreprises employant entre 500 et 2000 salariés y sont surreprésentées. Le client aurait, s'il se séparait de l'entreprise, un coût jugé relativement élevé de recherche d'un autre fournisseur. Mais cette dépendance tend à être réciproque. Les firmes de cette classe sont plutôt au nord du graphique 2 , relevant du monde marchand alors que la formation de la rentabilité est guidée par l'organisation de la production et la technologie, ce qui correspond au modèle industriel. L'optimisation met l'accent sur le moyen-long terme. L'intensité du capital (avec celle de la classe 3 ) et la productivité du travail y sont les plus fortes; mais la productivité du capital est dans la moyenne de l'échantillon et le coût moyen de la main-d'œuvre est le plus élevé. Les besoins en fonds de roulement sont également plus lourds. Ce dernier phénomène, imputable à la longueur des créances clients, correspond à la spécialisation dans des équipements destinés à des secteurs en aval particuliers. Il traduit les contraintes liées à la dédicace du produit, à des segments de demande et à des usages spécifiques. Cette classe 2 est également dépendante des actifs spécifiques (au sens de Williamson ${ }^{8}$ ) qu'elle a dû accumuler.

8. Actifs dédiés à des demandes particulières et donc «spécifiques ». Selon Williamson (1986), les investissements dédiés à des acheteurs particuliers, dans un contexte où l'opportunisme des acteurs économiques est présumé, font encourir un risque spécifique à l'entreprise qui les a réalisés. 


\section{Classe 3 (25,7\% des entreprises) : produits de base génériques}

La classe 3 regroupe des firmes "tayloriennes » : tâches répétitives, fabrication en grande série de produits considérés comme de milieu et bas de gamme et génériques. Assez logiquement, les dirigeants interrogés considèrent que la compétitivité de leur entreprise repose sur leur avantage en matière de prix. Les ventes aux grossistes constituent un débouché significatif. Cette classe se situe dans le quadrant sud-ouest du graphique 2. Elle apparaît comme l'archétype de la mise en cuvre du modèle de formation de la rentabilité guidée par l'organisation, caractérisé par une productivité du travail élevée, résultant d'une forte intensité capitalistique similaire à celle de la classe 2. En revanche, l'efficacité du capital est réduite. La formation de la rentabilité repose donc sur une organisation de la production favorisant les gains de productivité du travail.

Cette classe se distingue fortement de la suivante même si elles partagent une absence d'influence de la taille sur l'appartenance ou non à l'une d'entre elles. En effet, on constate une certaine concentration des firmes situées dans le secteur de la première transformation des matières premières alors que les sociétés composant la classe 4 relèvent plus fréquemment des secteurs de la mécanique.

\section{Classe 4 (25,4\% des entreprises) :}

\section{produits sur mesure, destinés à quelques clients du groupe}

La classe 4 rassemble des entreprises dont le chiffre d'affaires est réalisé majoritairement avec d'autres entreprises ou établissements qui leur sont liés. Les produits sont principalement de haut de gamme et faiblement standardisés. Plus de la moitié du chiffre d'affaires provient des quatre premiers clients et la production est plutôt en moyenne série. Si ces entreprises dépendent de quelques clients, la réciproque s'observe également, la spécificité des attentes des clients étant élevée. La compétitivité repose sur l'originalité et/ou la supériorité de l'offre. La rentabilité vient du produit, à condition que sa qualité soit reconnue par l'acheteur et que cette reconnaissance se traduise par un prix suffisant pour permettre la valorisation des actifs de l'entreprise. La qualité des produits, qui sont plutôt de haut de gamme, est en effet obtenue dans des conditions qui ne permettent d'optimiser ni la productivité du travail, ni le taux d'utilisation des équipements ou leur efficacité. Mais tant que cette qualité est reconnue par le marché, c'est-à-dire tant qu'elle répond aux attentes des clients, l'entreprise peut gérer sa contrainte de rentabilité en maîtrisant ses besoins en fonds de roulement, notamment par des délais de rotation des stocks faibles. Les entreprises de cette classe semblent fortement spécialisées, comme l'indique leur positionnement à l'est du graphique 2 . 
Classe 5 (20,6\% des entreprises): produits sur mesure, fabriqués à l'unité

La classe 5 rassemble des sociétés qui présentent des caractéristiques communes à celles de la classe 4. Elles s'en différencient, cependant, par l'importance des biens produits à l'unité, une moindre dépendance envers quelques clients et par une organisation du travail faisant appel à une certaine polyvalence des employés. Le poids des petites PME (moins de 100 salariés) est élevé, ainsi que celui des entreprises de la petite construction mécanique et électrique. On peut émettre l'hypothèse qu'il s'agit en grande partie de sous-traitants offrant des produits spécialisés dont les savoir-faire spécifiques correspondraient à la nécessité de s'adapter à l'incertitude particulièrement forte du marché. La classe 5 se situe au quadrant sud-est du graphique 2 .

Au total, les entreprises de la classe 1, et dans une certaine mesure celles de la classe 5 , gèrent, semble-t-il, leur rentabilité selon un système d'action qui serait guidé par le marché, alors que celles des classes 2 et 3 , surtout cette dernière, auraient une gestion guidée par l'organisation de la production. Les entreprises de la classe 4 peuvent plus difficilement être analysées en référence à ces modèles, même si, comme celles de la classe 5, elles semblent positionnées sur la recherche d'une spécialisation rentable. On relève, en outre, une certaine indépendance entre la taille de l'entreprise et l'appartenance à une classe, sauf pour les classes 2 et 5 .

En définitive, c'est avec les firmes à actifs spécialisés des classes 2, 4 et 5 que les banques devraient entrer dans un processus de compréhension de leur système d'action fondé sur le produit. La formation de leur rentabilité n'est pas réductible à une seule échelle de mesure. On ne peut se contenter de jugements ni ex post sur leurs ratios de rentabilité et / ou sur leur situation financière ni a priori sur la taille. L'analyse typologique montre la pluralité des «qualités» des entreprises selon la nature de leurs produits. Les ratios dits « interprétatifs » ne font sens que s'ils sont mis en cohérence. Associés à la connaissance des caractéristiques qualitatives des produits, ces ratios indiquent le mode de gestion de la rentabilité privilégié. Ces indicateurs renseignent sur la nature de la cohérence à gérer et, par conséquent, sur le type de risques à envisager.

\section{Une structure du capital plutôt homogène}

Dans une optique d'évaluation, l' analyse financière accorde traditionnellement une grande importance aux perspectives de rentabilité, mais aussi à la structure du capital des entreprises.

Les variables financières constituent, dans cette étude, des variables illustratives et non actives, c'est-à-dire qu'elles n'interviennent pas dans la définition des axes de l'analyse factorielle ni, par conséquent, dans la construction des classes d'entreprises. 
Le tableau en annexe montre que celles-ci ne se différencient pas selon le taux d'endettement. Le test d'inégalité des moyennes révèle, en effet, que ce dernier est similaire d'une classe à l'autre. L'effet discriminant que pourraient exercer d'autres variables financières (part des crédits bancaires courants, recours aux prêts des groupes et associés, coût du crédit, etc.) a été envisagé, mais aucune d'entre elles ne conduit à un résultat robuste, ce qui indique une relative homogénéité des structures financières, selon les classes.

Les correspondances attendues entre la structure de l'actif et celle du passif ne s'observent pas. Il va de soi que la structure des actifs dépend du positionnement de l'entreprise, c'est-à-dire de la nature du produit et des relations avec le marché. Les actifs immatériels spécialisés, par exemple, sont nécessaires à l'activité des entreprises dont les méthodes de production ne sont pas standard et qui répondent à une demande particulière, non répétitive (graphique 5). À la diversité de la structure de l'actif selon les classes correspondent diverses modalités de construction de la rentabilité, mais une relative homogénéité en matière d'autonomie financière. La pluralité des systèmes d'action des entreprises, dont rend compte l'analyse en classes, ne se retrouve donc pas lorsqu'on étudie leur structure financière. Les variables interprétatives «de la sphère réelle» différencient beaucoup plus les classes que les variables « de la sphère financière».

Nous avons pu constater que la taille des entreprises n'était pas un critère important dans l'analyse des différents types de cohérence " organisation et marché ». En outre, leur définition même d'une PME est difficile. Le critère des effectifs a l'avantage de la simplicité, mais l'inconvénient de l'arbitraire de la définition des seuils. La théorie financière n'oppose pas les sociétés selon leur taille, et les écarts, quand ils existent, résulteraient plutôt des conditions et de l'organisation de l'accès aux marchés financiers ${ }^{9}$, ce qui sort du champ de ce travail. En revanche, nous voulons insister sur l'hypothèse que le constat d'une certaine homogénéité des structures financières traduit l'absence de diversité des outils d'évaluation des performances des entreprises et non une indifférence des choix d'investissement au choix de financement.

La référence à une structure optimale du capital est au cœur de la théorie financière ${ }^{10}$. Sans vouloir reprendre toute la littérature sur ce sujet, rappelons le

9. De ce point de vue, une PME serait une entreprise non cotée sur un marché, la cotation étant un indicateur du degré d'accès à un financement non intermédié (Osteryoung et Newman, 1993) pour laquelle le seuil de 2000 salariés est une approximation (Cieply et Paranque, 1996).

10. Peu de travaux théoriques ou empiriques relient la structure du capital à la nature de l'activité économique de la firme. Pour une revue de la littérature, on peut lire Harris et Raviv (1991). On peut cependant citer l'approche de Williamson qui considère que les actifs standard ou redéployables doivent être financés par un contrat (standard) d'endettement, tandis que les actifs spécifiques doivent être financés par des ressources propres. 
théorème de Modigliani et Miller (1958) qui fonde la rationalité de la décision d'investissement en la dissociant de son mode de financement. Par la suite, de nombreux travaux ont cherché à construire une théorie de la structure optimale du capital en introduisant la notion de conflit d'intérêts entre «l'agent » et le «principal» et celle d'asymétrie d'informations entre les agents. Suivant l'approche mise de l'avant par Jensen et Meckling (1976), la structure du capital répond au souci de minimiser les coûts d'agence (ou coût des arrangements institutionnels mis en œuvre par les actionnaires et les créanciers qui conduit l'agent à maximiser la valeur de la firme). Suivant d'autres courants (Leland et Pyle, 1977), la structure du capital permet aux dirigeants d'adresser un signal de la qualité de la firme au marché financier. Dans cette perspective d'optimisation, la structure du capital constitue, bien entendu, un objectif.

Parmi l'abondante littérature sur ce sujet, les travaux de Myers et Majluf (1984) méritent une attention particulière. Leur approche, en effet, est quasiment la seule qui permette d'interpréter la relative homogénéité de la structure du capital d'une classe à l'autre, même si tel n'est pas l'objectif affiché par ces auteurs, leur souci étant d'expliciter l'importance de l'autofinancement. La structure du capital s'interprète en termes de hiérarchie des préférences ; les décisions de financement qui influencent les décisions d'investissement sont rationnelles dès lors que l'on admet que les dirigeants cherchent à maximiser la richesse des anciens actionnaires (corporate wealth). Selon cette construction, les dirigeants privilégient l'autofinancement et font appel au financement externe (l'endettement ayant la priorité) essentiellement pour financer des occasions d'investissement non prévues. La structure du capital observée est contingente puisqu'elle résulte des possibilités, incertaines, de financer des projets non prévus en faisant appel à des ressources externes (endettement, émission de capital). Les régularités observées concernant la structure du capital sont des résultats statistiques qui reflètent l'historique des besoins de financement externes et non l'expression d'un objectif de maximisation de la valeur de la firme.

Suivant Myers $(1977,1984)$, la rentabilité passée et présente, qui définit la capacité d'autofinancement, joue un rôle crucial dans la détermination de la structure du capital. Le taux de financement externe (y compris les émissions de capital) est d'autant plus faible que les entreprises ont été rentables et que les occasions d'investissement sont limitées. Cette interprétation permet de rendre compte de la tendance à l'homogénéité des structures du capital observées, pour une période et un secteur donnés, et du constat selon lequel les entreprises rentables s'autofinancent.

Cependant, il est possible d'analyser autrement la structure du capital, la perspective proposée ici n'étant pas construite en opposition, mais en complément 
de celle de Myers ${ }^{11}$. La préférence des dirigeants pour l'autofinancement puis pour l'endettement peut être interprétée en référence aux conventions de financement (Rivaud-Danset et Salais, 1992). Les conventions permettent aux acteurs de se coordonner. Cette problématique met en avant deux dimensions de la coordination. La première concerne la nature de l'incertitude que doivent traiter concrètement les acteurs concernés : les conventions de financement, c'est-à-dire les régimes de confiance qui peuvent s'établir entre l'entreprise et son environnement financier, diffèrent selon que l'incertitude peut être traitée (ou non) comme un risque probabilisable. La seconde est liée à la compatibilité des visées des dirigeants, des actionnaires et des banquiers : les conventions délimitent les choix organisationnels, les registres d'actions possibles. La structure du capital devient une norme acceptée par les parties, sur laquelle s'accordent les acteurs concernés ${ }^{12}$. Ces derniers s'entendent aussi sur un niveau de solvabilité en fin de période (lors du remboursement du prêt) qui correspond à une rentabilité économique ex ante (Fayolle et Fleurbaey, 1990) : c'est la rentabilité prévue qui guide les choix de financement.

Bien que le besoin de financement externe d'une entreprise ait toutes les raisons de varier (dans sa nature, ses échéances et son intensité, selon le modèle d'organisation et le produit), des forces tendent à une homogénéisation de la structure du capital ${ }^{13}$. Parmi les facteurs d'homogénéisation, l'un est relié à l'activité «réelle », soit la rentabilité et l'autre, à l'activité « financière », soit les normes de financement. Le taux d'endettement peut, suivant l'économie des conventions, s'analyser comme une norme associée au taux de rentabilité ex ante (capacité d'endettement) ou, suivant Myers, comme une variable dépendante du taux de rentabilité ex post (capacité effective d'autofinancement). Or le taux de rentabilité n'est pas un indicateur de la hiérarchie entre les modèles de produits. Ces derniers sont rentables s'ils sont mis en œuvre de façon cohérente. Et les tests statistiques ont montré que quatre des cinq classes d'entreprises présentent une rentabilité équivalente. Si l'on admet que les financements ne sont pas substituables, en particulier pour les PME, et que les possibilités d'endettement d'une entreprise s'apprécient par référence à des seuils, il n'est pas étonnant que la dispersion de la structure du capital soit limitée. Ainsi,

11. L'hypothèse, commune, d'asymétrie d'informations nous conduit à considérer que les acteurs ne peuvent pas, dans toutes les situations, décider selon le principe de la rationalité substantive. Dans de nombreuses situations, qui intéressent particulièrement l'économie des conventions, l'incertitude n'est pas probabilisable, au sens où il n'existe pas de probabilité fréquentiste et où les acteurs ne peuvent envisager toutes les occurrences futures, de sorte que son traitement dépend d'un exercice de jugement (Knight, 1921).

12. Ces normes d'endettement, qui évoluent dans le temps et dans l'espace, sont sensibles au rationnement du crédit par les quantités et au niveau des taux d'intérêt.

13. La référence au système national de financement prédit une faible dispersion des données individuelles concernant la structure du capital d'entreprises résidant dans un pays et à une période donnés. 
l'homogénéité relative de la structure du capital observée empiriquement illustre, dans notre perspective, les difficultés des banques à différencier les entreprises selon la nature de leur produit, bien que les besoins de financement varient en fonction de cette nature, telle qu'elle a été définie ici. La variété plus ou moins grande des structures de financement dépendra de la façon dont les apporteurs de capitaux construisent et traitent l'information, de leur capacité à disposer d'outils d'analyse du risque différents non seulement selon la taille des entreprises, mais aussi selon leur «qualité » fondée sur ce que nous avons appelé un système d'action.

La limitation des choix et des conventions de financement réduit les possibilités de déplacement des firmes vers d'autres systèmes d'action. Si, en cas de chute de la rentabilité, une entreprise modifie son système d'action en réaction à ses difficultés, les changements de la structure de son passif et des conditions de formation de sa rentabilité peuvent être interprétés par les offreurs de capitaux, en particulier les banques, non pas comme un déplacement susceptible de lui permettre de restaurer sa rentabilité, mais comme le signe d'une aggravation de ses difficultés. La norme induite par la convention de financement peut se transformer en une contrainte qui apparaît alors imposée de l'extérieur et limite les possibilités de déplacement. Dans ce cas, la gestion du risque, tant par les banques que par les autres partenaires, peut provoquer des perturbations supplémentaires, voire précipiter l'échec. Un déplacement vers un autre système d'action suppose, pour réussir, que l'entreprise puisse s'appuyer sur une diversité d'outils d'analyse de la part des tiers.

\section{Des firmes différenciées et des rentabilités comparables: un éclairage théorique}

Les modèles de formation de la rentabilité et les résultats de l'analyse de données ont confirmé qu'il existe plusieurs façons d'être rentable. Cette dernière partie se propose de commenter un autre résultat. Les entreprises des classes 1 et 2 , malgré un taux élevé de dépenses immatérielles (marketing, recherche et développement), ne réalisent pas une rentabilité économique plus importante que celle des firmes des autres classes.

On se propose donc ici de rechercher dans la littérature théorique l'effet que peuvent exercer sur la formation de la rentabilité les ressources spécialisées d'une entreprise ${ }^{14}$. Dans cette perspective, nous nous orientons, dans un premier temps, vers Marshall puisqu'il a introduit la notion de quasi-rente, puis vers la théorie évolutionniste qui est particulièrement soucieuse de rendre compte de la diversité des firmes.

14. En utilisant l'expression « ressources spécialisées », nous avons volontairement cherché à distinguer ces ressources des actifs dédiés à des demandes particulières et donc «spécifiques» au sens de Williamson. 


\section{GraPhiQue 5}

Taux de dépenses immatérielles (en pourcentage)

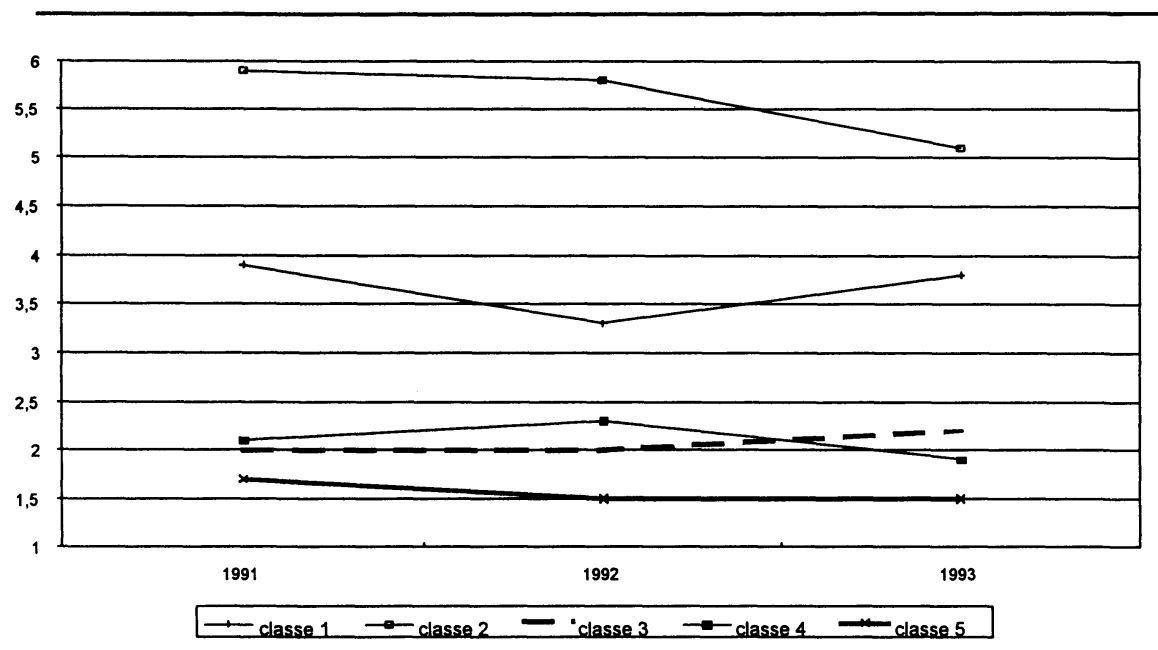

Source : Banque de France.

Marshall, dans les Principles of Economics a introduit la notion de quasi-rente et, en outre, a évoqué celle de capital immatériel qu'il désigne comme les relations d'une entreprise (business connection) ${ }^{15}$. La quasi-rente, qui découle d'un avantage différentiel, se définit comme un revenu déterminé par l'état du marché qui permet au prix de ne pas être déterminé directement par les coûts normaux de production et le profit normal. Mais, précise Marshall dans une note destinée à expliciter la quasi-rente (V, IX, 5), ce profit n'est «non nécessaire » qu'à court terme. Dans le long terme, il devient nécessaire pour compenser les coûts qui s'ajouteront aux coûts initiaux qui devront être assumés par l'entreprise. Prévoir, avec confiance, de futures quasi-rentes est indispensable pour inciter à investir des capitaux dans des équipements qui impliqueront des coûts supplémentaires, parfois très élevés au regard de la dépense initiale, comme l'illustre le cas du télégraphe sous-marin cité en exemple par Marshall. La quasi-rente peut donc être considérée comme un gain d'opportunité de court terme offrant la perspective d'un taux normal de profit dans

15. Pour Marshall, c'est l'ensemble des perspectives de gain concourant à former le profit à long terme qui incite à entreprendre. Les investissements en capitaux et en énergie personnelle pour mettre en place les dispositifs pour la production future et pour construire le capital «immatériel » d'une entreprise doivent apparaître comme étant susceptibles d'être rentables avant d'être engagés (Marshall, 1920, p. 618). 
le long terme. La quasi-rente cumulée s'analyse alors comme la contrepartie des dépenses nécessaires à long terme au maintien ou à l'amélioration de l'activité. La théorie marshallienne de la firme ne permet donc pas de dégager les fondements d'une rentabilité supérieure en longue période à celle de l'entreprise représentative dont le taux de profit est normal, et qui seraient distincts d'une rente de situation ou de rareté.

Les théories contemporaines tendent à abandonner la firme représentative marshallienne. Un premier pôle de ces théories est représenté par les économistes évolutionnistes. Préoccupés d'introduire la diversité, ils ont mis l'accent sur l'importance des routines et des savoir-faire organisationnels et technologiques acquis par la firme par apprentissage interne (learning by doing and by using). Ces ressources spécialisées, dont l'entreprise s'est dotée progressivement, sont souvent tacites, idiosyncrasiques, donc non transférables (non redéployables) hors de l'entreprise, et non quantifiables directement puisqu'elles ne trouvent pas leur origine dans des dépenses clairement identifiables. Les évolutionnistes, préoccupés par la sélection, considèrent que la force compétitive et la viabilité d'une entreprise dépendent de «sa compétence foncière » et de ses relations avec ses clients et ses fournisseurs. Une «compétence foncière" se définit comme «un ensemble de compétences technologiques différenciées, d'actifs complémentaires et de routines qui constituent la base des capacités concurrentielles d'une entreprise dans une activité particulière » (Dosi, Teece et Winter, 1990) ${ }^{16}$. Par rapport à notre problématique, nous remarquons que la compétence foncière, qui caractérise une firme et lui donne un avantage spécifique en matière de compétitivité, est, certes, source de différenciation entre les firmes, mais elle ne conduit pas à fonder théoriquement l'existence d'une hiérarchie des performances.

L'approche financière de l'entreprise constitue un autre pôle des recherches contemporaines. Les compétences tacites sont traitées comme des actifs; les ressources spécialisées peuvent être source de survaleur. La survaleur (business goodwill), qui se déduit d'un écart positif observé sur plusieurs années entre la valeur de la firme par rapport à la rentabilité moyenne du capital, rend compte des actifs incorporels non directement quantifiables, mais générateurs de bénéfices. Fondamentalement, la méthode utilisée pour évaluer les actifs incorporels présuppose qu'il n'existe pas une hiérarchie de rentabilité entre firmes, puisque le constat d'un taux de profit plus élevé conduit à réévaluer le dénominateur en y intégrant des actifs incorporels, en particulier l'efficience organisationnelle de la firme et la qualification de la main-d'œuvre. «Ce que la firme sait faire» s'ajoute à ses équipements.

16. L'ouvrage de Coriat et Weinstein présente de façon synthétique la théorie de la firme produite par les auteurs évolutionnistes (Coriat et Weinstein, 1995). 


\section{Conclusion}

En raison de la pluralité des «qualités » des entreprises, un même ratio synthétique de rentabilité peut exprimer des réalités très différentes ${ }^{17}$. Aussi, pour évaluer correctement les performances des firmes, il est nécessaire d'associer à la connaissance des caractéristiques qualitatives des produits l'examen de ratios dits « interprétatifs » qui indiquent les modes de gestion de la formation de la rentabilité. Suivant la problématique, très répandue, de conseils en stratégie qui privilégient le diagnostic des points forts et des points faibles, il est souvent recommandé de se débarrasser de ses points faibles. Or les «points faibles» de ces entreprises sont justement ce qui peut permettre à leurs «points forts » de s'exprimer.

L'évaluation d'une firme et l'interprétation de ses ratios par des tiers supposent donc une lisibilité accrue, en particulier grâce à des informations qualitatives. Parmi ces données, l'accent doit être mis sur les relations de l'entreprise avec son environnement, en particulier financier, afin :

- d'identifier les modalités de financement, entre autres, dans le cadre des relations de groupe, et la façon dont est assurée la pérennité ;

- de comprendre si un faible ratio d'endettement indique un rationnement du crédit subi ou choisi ;

- de comprendre si les délais de règlement avec les fournisseurs et les clients sont coutumiers ou traduisent des difficultés conjoncturelles.

La diversité modérée de situations mises en évidence par la typologie renforce le besoin d'une lecture de la performance qui puisse en rendre compte, donc qui prenne appui sur la compréhension de la manière dont cette performance est concrètement obtenue par le système d'action de l'entreprise : l'approche devient procédurale. En d'autres termes, un ratio ne doit pas être analysé à partir d'une norme fixée a priori, mais plutôt selon les conditions de sa réalisation. Plus il est synthétique, plus la procédure sous-jacente doit être identifiée. Partant de là, l'appréciation du ratio ne peut plus se faire de manière binaire, mais du point de vue de la cohérence du processus économique correspondant (Salais et Storper, 1993; Paranque, 1994, 1995), de sorte que le jugement normatif formulé par les tiers, offreurs de capitaux, est fixé à l'issue d'une délibération procédurale où la cohérence du système d'action est privilégiée et non une norme a priori et indifférenciée. L'entreprise doit pouvoir

17. Ainsi, un processus peu formalisé d'essais et d'erreurs est source d'économies en cas de succès, mais aussi de surcoûts et donc de pertes en cas d'échec. Les compétences acquises par la répétition sont porteuses de contraintes particulières en matière de rentabilité. Leur maintien suppose une certaine stabilité dans le temps de la relation de travail, ce qui limite les ajustements de l'emploi aux variations du niveau de l'activité et se traduit par une sensibilité élevée de la productivité apparente du travail à la conjoncture. 
donner à lire à son partenaire financier la cohérence multidimensionnelle de son action, et ainsi permettre au prêteur d'améliorer la qualité de son propre actif. Dans certains cas, cela conduira à «déqualifier» l'entreprise par rapport à ce que donnerait la lecture automatique de ses ratios ; dans d'autres cas, à la « requalifier » et ainsi donner une qualité plus élevée et plus fiable aux actifs correspondants de son partenaire financier.

La difficulté d'évaluation par les tiers de savoir-faire spécifiques qui ne laissent aucune trace au bilan et le caractère non redéployable à d'autres produits des actifs spécifiques (au sens de Williamson [1986]) ou de biens dédiés à des demandes particulières, même s'ils sont matériels, incitent les apporteurs de capitaux externes, notamment les banques, à rationner ce type d'entreprises qui manquent de garanties. La disponibilité de données élaborées d'ordre qualitatif ou subjectif (de ce fait, difficilement transmissibles et accessibles à des tiers) améliorerait sensiblement la lisibilité des PME. Ces données, dès lors qu'elles seraient jugées pertinentes par toutes les instances décisionnelles, sécuriseraient les banques ainsi que l'ensemble des partenaires, réduiraient la déperdition de connaissances qui se produit dans la circulation administrative des dossiers. Une évaluation fondée sur la «pluralité » des qualités d'entreprises permettrait de mieux repérer les tensions, les sources de fragilité spécifiques à un système d'action et d'en étudier les remèdes. Elle pourrait ainsi permettre à des entreprises hors normes d'accéder plus aisément au marché du crédit.

Une lecture de ces informations, qui met l'accent sur la cohérence entre les modalités d'organisation de la production et les relations des entreprises avec leur environnement, permettrait également d'améliorer les capacités de prévision. Un même ratio synthétique de rentabilité peut, nous l'avons vu, exprimer des réalités très différentes et, de ce fait, une sensibilité à la conjoncture très inégale qui devrait être intégrée à la prévision. Or celle-ci n'est pas la même selon que l'entreprise a, par exemple, quelques clients particuliers ou un grand nombre et, plus généralement, elle differe selon la nature de son produit. Ainsi, les gains d'opportunité envisagés la quasi-rente marshallienne - incitent des entreprises à se spécialiser dans des produits soumis à une forte incertitude. Cette approche soulève pour l'observateur une question qu'une lecture de Marshall pourrait éclairer. L'importance de la dynamique chez ce théoricien nous rappelle que ce que l'on observe est en coupe, et même si l'observation concerne plusieurs années, l'observateur ne sait pas où l'entreprise se situe dans la dynamique. La distinction introduite par Marshall entre les différentes périodes de temps, du très court terme au très long terme, souligne les limites de toute observation des performances des entreprises réalisée sur une période donnée. L'observateur court en effet le risque de ne pas saisir la dynamique de long terme qui lui permettrait d'interpréter de façon satisfaisante un résultat qui s'écarte de la moyenne. Ainsi, un déficit ne sera que conjoncturel si des dépenses nécessaires à l'amélioration de l'activité n'auront pas encore produit 
leur plein effet et une performance supérieure à la moyenne ne sera qu'une quasirente marshallienne dans le cas où ces mêmes dépenses n'auront pas encore été entreprises.

\section{Bibliographie}

Bardos, M. et B. PARANQue (1992), Des trajectoires dans la transformation du tissu industriel, Centrale de bilans, Banque de France.

CIEPly, S. et B. PARANQue (1996), Comportement d'endettement des entreprises industrielles françaises sur la période 1990-1993, Banque de France, T 96/02.

CORIAT, B. et O. WEINSTEIN (1995), Les nouvelles théories de l'entreprise, Paris, Le livre de poche.

Dosi, G., D.J. TEECE et S.G. WINTER (1990), «Les frontières des entreprises : vers une théorie de la cohérence de la grande entreprise », Revue d'Économie Industrielle, $1^{\text {er }}$ trimestre, $n^{0} 51$, p. 238-257.

Fayolle, J. et M. Fleurbaey (1990), «Accumulation, profitabilité et endettement des entreprises », Document de travail, INSEE.

HARRIS, M. et A. RAVIV (1991), «The theory of capital structure», Journal of Finance, vol. 46, 0-32, $\mathrm{n}^{\circ} 1$, mars.

JENSEN, M.C. et W. MECKLING (1976), «Theory of the firm : managerial behavior, agency costs and ownership structure », Journal of Financial Economic, octobre.

KNight, F.H. (1921), Ride, Uncertainty and Profit, Boston, Houghton Mifflin Company.

LELAND, H. et D. PYLE (1977), «Information asymetrics, financial structure and financial intermediation ", Journal of Finance, vol. 32.

Marshall, A. (1920), Principles of Economics, $8^{e}$ édition, réimprimé en 1994, Londres, Macmillan.

Modigliani, F. et M.H. MiLleR (1958), «The cost of capital, corporate finance and the theory of investment ", American Economic Review, mai.

MYERS, S.C. (1977), «Determinants of corporate borrowing », Journal of Financial Economics, $\mathrm{n}^{\circ} 2$, juillet.

MYERS, S.C. (1984), «The capital structure puzzle », Journal of Finance, vol. 39, juillet.

MYERS, S.C. et N.S. MAJLUF (1984), « Corporate financing and investment, decision when firms have information that you do not have », Journal of Financial Economics, juillet. 
OSTERYOUNG, J.S. et D. NEWMAN (1993), «What is a small business », The Journal of Small Business Finance, vol. 2, $\mathrm{n}^{\circ} 3$, p. 219-231.

PARANQUe, B. (1994), Emploi, accumulation, rentabilité financière, Banque de France, Collection Entreprises, B 94/02.

PARANQue, B. (1995), Compétitivité et rentabilité des entreprises industrielles, Banque de France, Collection Entreprises, B 95/06.

PARANQue, B., D. Rivaud-DANSET et R. SAlais (1996), « Marchés, organisation de la production et rentabilité : une exploitation de la base de données stratégiques de la Banque de France », Banque de France, Les Études de l'Observatoire des entreprises, T 96/03.

RIVAUD-DANSET, D. et R. SALAIS (1992), « Les conventions de financement des entreprises », Revue Française d'Économie, vol. 2, no 4.

SALAIS, R. et M. STORPER (1993), Les mondes de production, Enquête sur l'identité économique de la France, Paris, Édition de l'École des hautes études en sciences sociales.

Williamson, O.E. (1986), Economic, Organization, Firms, Market and Policy Control, Boston, Wheatsheafbooks. 
Quelques statistiques sur les classes d'entreprises*

\begin{tabular}{|c|c|c|c|c|c|c|}
\hline \multirow[b]{2}{*}{ Classe } & \multicolumn{3}{|c|}{$\begin{array}{c}\text { Valeur ajoutée globale/ } \\
\text { effectifs }(K F / p)\end{array}$} & \multicolumn{3}{|c|}{$\begin{array}{l}\text { Taux de marge brute } \\
\text { globale (\%) }\end{array}$} \\
\hline & $\begin{array}{c}\text { Borne } \\
\text { inférieure }\end{array}$ & Moyenne & $\begin{array}{l}\text { Borne } \\
\text { supérieure }\end{array}$ & $\begin{array}{c}\text { Borne } \\
\text { inférieure }\end{array}$ & Moyenne & $\begin{array}{l}\text { Borne } \\
\text { supérieure }\end{array}$ \\
\hline 1 & 238,0 & 251,8 & 269,7 & 7,8 & 9,3 & 10,7 \\
\hline 2 & 287,7 & 300,9 & 314,2 & 8,2 & 9,2 & 10,3 \\
\hline 3 & 278,3 & 289,6 & 300,8 & 8,8 & 9,7 & 10,6 \\
\hline 4 & 247,4 & 258,8 & 270,2 & 8,3 & 9,2 & 10,1 \\
\hline 5 & 240,1 & 252,7 & 265,3 & 4,9 & 5,9 & 7,0 \\
\hline & \multicolumn{3}{|c|}{$\begin{array}{l}\text { Délai de rotation des besoins } \\
\text { en fonds de roulement (jours) }\end{array}$} & \multicolumn{3}{|c|}{$\begin{array}{c}\text { Taux de rotation } \\
\text { des équipements productifs (\%) }\end{array}$} \\
\hline Classe & $\begin{array}{c}\text { Borne } \\
\text { inférieure }\end{array}$ & Moyenne & $\begin{array}{l}\text { Borne } \\
\text { supérieure }\end{array}$ & $\begin{array}{c}\text { Borne } \\
\text { inférieure }\end{array}$ & Moyenne & $\begin{array}{c}\text { Borne } \\
\text { supérieure }\end{array}$ \\
\hline 1 & 85,4 & 94,8 & 104,2 & 317,9 & 371,4 & 424,9 \\
\hline 2 & 101,5 & 108,4 & 115,4 & 296,7 & 336,4 & 376,0 \\
\hline 3 & 79,2 & 85,1 & 91,0 & 241,6 & 275,2 & 308,9 \\
\hline 4 & 76,7 & 82,7 & 88,7 & 204,8 & 238,9 & 273,1 \\
\hline 5 & 59,6 & 66,2 & 72,8 & 363,4 & 401,0 & 438,6 \\
\hline & \multicolumn{3}{|c|}{ Frais de personnel/effectifs (KF/p) } & \multicolumn{3}{|c|}{ Taux de dépenses immatérielles } \\
\hline Classe & $\begin{array}{c}\text { Borne } \\
\text { inférieure }\end{array}$ & Moyenne & $\begin{array}{c}\text { Borne } \\
\text { supérieure }\end{array}$ & $\begin{array}{c}\text { Borne } \\
\text { inférieure }\end{array}$ & Moyenne & $\begin{array}{l}\text { Borne } \\
\text { supérieure }\end{array}$ \\
\hline 1 & 179,2 & 186,6 & 194,1 & 2,8 & 3,8 & 4,8 \\
\hline 2 & 216,8 & 222,3 & 227,9 & 4,4 & 5,1 & 5,9 \\
\hline 3 & 198,8 & 203,5 & 208,2 & 1,2 & 1,9 & 2,5 \\
\hline 4 & 190,9 & 195,7 & 200,4 & 1,6 & 2,2 & 2,9 \\
\hline 5 & 206,0 & 211,2 & 216,5 & 0,8 & 1,5 & 2,2 \\
\hline & \multicolumn{3}{|c|}{ Taux d'endettement } & \multicolumn{3}{|c|}{ Délais de paiement clients (jours) } \\
\hline Classe & $\begin{array}{c}\text { Borne } \\
\text { inférieure }\end{array}$ & Moyenne & $\begin{array}{l}\text { Borne } \\
\text { supérieure }\end{array}$ & $\begin{array}{c}\text { Borne } \\
\text { inférieure }\end{array}$ & Moyenne & $\begin{array}{l}\text { Borne } \\
\text { supérieure }\end{array}$ \\
\hline 1 & 46,2 & 54,8 & 63,4 & 80,0 & 84,7 & 89,4 \\
\hline 2 & 38,7 & 45,1 & 51,5 & 92,6 & 96,0 & 99,5 \\
\hline 3 & 38,9 & 44,3 & 49,8 & 80,6 & 83,6 & 86,5 \\
\hline 4 & 42,8 & 48,3 & 53,9 & 85,3 & 88,3 & 91,2 \\
\hline 5 & 43,6 & 49,6 & 55,7 & 91,3 & 94,6 & 97,9 \\
\hline & \multicolumn{3}{|c|}{$\begin{array}{l}\text { Délais de règlement } \\
\text { fournisseurs (jours) }\end{array}$} & \multicolumn{3}{|c|}{$\begin{array}{l}\text { Délais de rotation } \\
\text { des stocks (jours) }\end{array}$} \\
\hline Classe & $\begin{array}{c}\text { Borne } \\
\text { inférieure }\end{array}$ & Moyenne & $\begin{array}{c}\text { Borne } \\
\text { supérieure }\end{array}$ & $\begin{array}{c}\text { Borne } \\
\text { inférieure }\end{array}$ & Moyenne & $\begin{array}{c}\text { Borne } \\
\text { supérieure }\end{array}$ \\
\hline 1 & 71,3 & 75,7 & 80,2 & 61,1 & 68,5 & 75,8 \\
\hline 2 & 76,1 & 79,4 & 82,7 & 69,4 & 74,8 & 80,3 \\
\hline 3 & 73,4 & 76,2 & 79,0 & 50,7 & 55,3 & 59,9 \\
\hline 4 & 77,6 & 80,5 & 83,3 & 51,0 & 55,7 & 60,4 \\
\hline 5 & 88,4 & 88,6 & 91,7 & 57,8 & 63,0 & 68,1 \\
\hline
\end{tabular}

Source: Banque de France. 